\title{
Crio-fixation of Pachytene Cells
}

\author{
R. Ortiz-Hernandezı, G. H. Vázquez-Nin1, O. Echeverría-Martínez1, C. Höög2, A. \\ Hernández-Hernández3*. \\ 1Laboratorio de Microscopía Electrónica, Facultad de Ciencias, UNAM, México \\ ${ }_{2}$ Department of Cell and Molecular Biology, Karolinska Institutet, Stockholm \\ 3Laboratorio de Investigación en Patología Experimental, Hospital Infantil de México \\ Federico Gómez, México \\ *Corresponding author: abrahan.h.hernandez@gmail.com
}

\begin{abstract}
Genetic variability in organisms with sexual reproduction is produced by a complex mechanism of cell division of the germ line cells known as meiosis. During meiosis, homologous chromosomes pair and exchange genetic material (meiotic recombination), process that is essential to complete the meiotic division and to produce variability. Homologous chromosome pairing is mediated by the synaptonemal complex (SC). The SC is a proteinaceous structure composed of two lateral elements (LEs) and a central region (CR). Any defect in SC structure impairs meiotic recombination leading to blockage of the cell division process and infertility 1. The SC has been observed since the introduction of the electron microscope (EM) in the biological field and it has been reported to be present in almost all the organisms with sexual reproduction, conserving a very similar structure and organisation along the different species2,3. The classic approach to study the SC structure is to chemically fixate the sexual tissues (gonads), dehydrate and embedded them in plastic resins that provide a support for sample sectioning and observation under the EM. However, chemical fixation followed by dehydration is well known to produce artefacts in the structure of many biological samples. Recently, we have combined fluorescence activated cell sorting of cells with SCs with high-pressure freezing and freezesubstitution and have found that the structure of the CR looks different from that observed in chemically fixated samples. These data have prompted us to analyse the organization of the CR components under cryo-processing.
\end{abstract}

References:

[1] M.A. Handel, J.C. Schimenti, Nat Rev Genet. 11(2) (2010) p. 124-36.

[2] M.J. Moses, J Biophys Biochem Cytol. 2(2) (1956) p. 215-8.

[3] J. Fraune, S. Schramm, Alsheimer, R. Benavente, Exp Cell Res. 318(12) (2012) p. 1340-6. 